\title{
Expression of autophagy-associated proteins in rat dental irreversible pulpitis
}

\author{
SHENGCAI QI ${ }^{1 *}$, JUN QIAN $^{2 *}$, FUBO CHEN $^{1 *}$, PENG ZHOU $^{2}$, JING YUE $^{1}$, FENGQIN TANG $^{1}$, \\ YIMING ZHANG ${ }^{1}$, SHIQIANG GONG ${ }^{3}$, GUANGWEI SHANG ${ }^{1}$, CHUN CUI $^{3}$ and YUANZHI XU ${ }^{1}$ \\ ${ }^{1}$ Department of Stomatology, Shanghai Tenth People's Hospital, Tongji University School of Medicine, Shanghai 200072; \\ ${ }^{2}$ Jiangsu Key Laboratory of Oral Diseases, Affiliated Hospital of Stomatology, Nanjing Medical University, Nanjing, \\ Jiangsu 210029; ${ }^{3}$ Center of Stomatology, Tongji Hospital, Tongji Medical College, \\ Huazhong University of Science and Technology, Wuhan, Hubei 430030, P.R. China
}

Received May 20, 2018; Accepted January 25, 2019

DOI: $10.3892 / \mathrm{mmr} .2019 .9944$

\begin{abstract}
Autophagy serves an important role in numerous diseases, as well as in infection and inflammation. Irreversible pulpitis (IP) is one of the most common inflammatory endodontic diseases, and autophagy has been reported to regulate IP in vitro. However, the level of autophagy in the IP pathogenic process in vivo remains unknown. The aim of the current study was, thus, to investigate the levels of autophagy-associated proteins in rats with IP in vivo. A rat dental IP model was successfully constructed, and five different time points $(0,1,3,5$ and 7 days) were investigated. The levels of the autophagy-related 5 (ATG5), ATG7, light chain 3 (LC3) and Beclin-1 proteins exhibited a time-dependent increase in rats with IP, whereas the levels of mammalian target of rapamycin and p62/sequestosome 1 were decreased. In addition, the levels of ATG proteins were specifically increased in odontoblasts and microvascular endothelial cells in pulpitis tissue. Based on these findings, autophagy may serve an important role in IP, and the present study data provide a new insight into the IP pathogenesis and treatment.
\end{abstract}

\section{Introduction}

Tooth pulp inflammation is also known as pulpitis, including reversible pulpitis and irreversible pulpitis (IP), and is one of

Correspondence to: Dr Chun Cui, Center of Stomatology, Tongji Hospital, Tongji Medical College, Huazhong University of Science and Technology, 1095 Jie Fang Avenue, Wuhan, Hubei 430030, P.R. China E-mail: pulpcapping@163.com

Dr Yuanzhi Xu, Department of Stomatology, Shanghai Tenth People's Hospital, Tongji University School of Medicine, 301 Yanchang Road, Shanghai 200072, P.R. China

E-mail: amyxyz01@hotmail.com

*Contributed equally

Key words: autophagy, irreversible pulpitis, rat dental pulpitis model the most common oral diseases. IP usually causes severe pain in patients, and root canal is the main method used to treat IP in endodontics. The dental pulp is particularly susceptible to ischemic conditions, and IP results in hypoxia and starvation (1). In addition to ischemia, IP is caused by microbial infections, predominately from anaerobic bacteria (2).

Toll-like receptors (TLRs) mediate signaling following stimulation with bacterial cell wall components during inflammatory reactions, which assist the host immune system by inducing inflammatory signaling pathways and producing inflammatory cytokines (3). Among the 13 TLRs (TLR1 to TLR13), TLR2 is mainly expressed during the early stage of pulp inflammation triggered by a bacterial infection (4), while TLR9 is upregulated by infection and tissue damage $(5,6)$. In addition, the transcription factor nuclear factor $-\kappa \mathrm{B}(\mathrm{NF}-\kappa \mathrm{B})$ regulates the inflammatory reaction and is activated by inflammation. NF- $\kappa \mathrm{B} 1$ is a DNA binding subunit of the NF- $\kappa \mathrm{B}$ protein complex and is expressed at high levels in lipopolysaccharide (LPS)-stimulated dental pulp cells (7). Therefore, TLRs and $\mathrm{NF}-\kappa \mathrm{B} 1$ are widely used as markers of inflammation.

Autophagy is an evolutionarily conserved cellular process that maintains cytoplasmic homeostasis (8). It is initiated with the formation of a lipidic double membrane vacuole known as the phagophore, and then the vacuole is elongated to form a mature autophagosome that engulfs cytoplasmic constituents. Subsequently, the autophagosome fuses with the lysosome to degrade the cargo, and the degraded component is finally released and recycled to maintain cellular hemostasis. Two ubiquitin-like conjugation systems, namely the autophagy-related 5-12 (ATG5-12) complex and light chain 3 (LC3; also termed the microtubule-associated protein 1 light chain 3 or ATG8), are associated with membrane elongation and autophagosome formation. ATG12 interacts with ATG5 to form the ATG5-ATG12 complex via ATG7, and the two systems require ATG7 for activation (8). Beclin-1 is part of the class III phosphatidylinositol 3-kinase complex required for the localization of ATGs to the phagophore and is located in the autophagosome $(8,9)$. In addition, mammalian target of rapamycin (mTOR) is known to be a key negative regulator of autophagy (10). Therefore, ATGs, LC3, mTOR and Beclin-1 are frequently used as autophagosome markers (10). Furthermore, 
p62/sequestosome 1 (p62/SQSTM1), an autophagy receptor for ubiquitin-like substrates in the phagophore $(11,12)$, serves as a marker of autophagic flux.

Autophagy has a positive effect in numerous inflammatory diseases, such as lung inflammation (13), tuberculosis and Crohn's disease (14).In these diseases, inflammation is controlled by the removal of aggregated inflammatory components through autophagy (15), which affects inflammation-associated signaling pathways. Autophagy provides a series of barriers for invading microorganisms, and inflammation is improved following the elimination of the pathogens (16). In addition, autophagy serves a critical role in maintaining the energy supply during stress, starvation and hypoxia (17). IP usually causes pulp stress, hypoxia and nutrition depletion resulting from a bacterial invasion, and may activate autophagy during the pathogenesis of this disease. In response to these bacteria, the disease activates the innate and adaptive immune systems of the host (18). Microbial infection and the immune response are tightly connected via autophagy (19). Furthermore, hypoxic and inflammatory environments also induce angiogenic processes (20), cell proliferation or cell protection through autophagy to help restore dental pulp cell homeostasis (21). Therefore, research on the role of autophagy in the process of IP would be of great importance.

A recent study has reported that autophagy negatively affected inflammation in dental pulpitis cells in vitro by regulating odontoblast differentiation (7). However, the immune environment in vivo is more complex in comparison with the in vitro environment, and autophagy activation in IP in vivo remains unknown. With the development of molecular imaging techniques, biomarker detection in vivo using fluorescence has been widely used (22-24), while the detection of fluorescent proteins is useful for biomarker analyses $(25,26)$. In the present study, the aim was to investigate the level of autophagy in IP in vivo and to analyze this using a fluorescence detection method. The findings of the study provide novel insights into the etiology and treatment of IP.

\section{Materials and methods}

Experimental animals. All experimental procedures were approved by the Committee on the Animal Care and Use of Laboratory Animals of the Shanghai Tenth People's Hospital, Tongji University School of Medicine (Shanghai, China). All experiments were performed on 4-week-old male Sprague-Dawley rats weighing 250-350 g. In total, 20 rats were used in the present study. The animals were all specific-pathogen free, and had free access to food in a clean, temperature-controlled room $\left(23^{\circ} \mathrm{C}\right)$ with a 12 -h light/dark cycle.

Preparation of the IP rat model. Each rat was anesthetized with an intraperitoneal injection of chloral hydrate at a concentration of $0.1 \mathrm{mg} / \mathrm{ml}$ and use of $1 \mathrm{ml}$ per $100 \mathrm{~g}$ body weight. Following anesthesia, rats were placed on a warm mat $\left(37^{\circ} \mathrm{C}\right)$ in supine position for surgery. The mouths of the rats were gently opened with metal tweezers, and the left maxillary first molars were drilled with a high-speed handpiece and a Dia-Bur $^{\circledR}$ (cat. no. BR-49; MANI, Inc., Utsunomiya, Japan) under water cooling. Fine paper points were then used to dry the tooth, and then the dental pulp was carefully inspected and opened using size \#15, length $25 \mathrm{~mm}$ K-files (MANI, Inc.), which caused the tooth pulp to bleed. The pulp cavity was exposed for 7 days, and then the rats were sacrificed according to the experimental design. Rats sacrificed immediately after surgery were designated to the control group (D0), which exhibited normal dental pulp tissue.

Hematoxylin and eosin $(H \& E)$ staining for rat IP. On days $0,1,3,5$ and 7 post-surgery (D0, D1, D3, D5 and D7, respectively), rats ( $n=4 /$ time point) were decapitated, and the molar tooth specimens were rapidly removed. Samples were fixed with $4 \%$ paraformaldehyde overnight at $4{ }^{\circ} \mathrm{C}$, followed by demineralization with $10 \%$ ethylenediaminetetraacetic acid ( $\mathrm{pH}$ 7.4) for 2 months at $4^{\circ} \mathrm{C}$. Following dehydration and paraffin embedding, the samples were sectioned into $5-\mu \mathrm{m}$ slices. Each section included coronal and radicular pulpitis tissue. Subsequent to drying for $2 \mathrm{~h}$ in an oven maintained at $55-60^{\circ} \mathrm{C}$, slices were dipped in xylene to remove the paraffin and rehydrated using a decreasing alcohol gradient. Next, the $5-\mu \mathrm{m}$ tooth tissue sections were washed with water for $5 \mathrm{~min}$ and stained with hematoxylin for $5 \mathrm{~min}$, followed by eosin for $5 \mathrm{sec}$. The stained slices were then washed, dehydrated and clarified in xylene. Finally, the sections were sealed with a fat-soluble gel and observed under a microscope.

\section{Immunohistochemical staining for TLR2, TLR 9 and NF- $\kappa B 1$} in the rat IP model. Subsequent to dewaxing and rehydration through an alcohol gradient, slices were treated with $3 \%$ $\mathrm{H}_{2} \mathrm{O}_{2}$ to block the endogenous peroxidases for $15 \mathrm{~min}$ at $37^{\circ} \mathrm{C}$, and antigen retrieval was conducted with $0.25 \%$ pancreatic enzymes for $10 \mathrm{~min}$ at $37^{\circ} \mathrm{C}$. The slices were then blocked with 5\% normal goat serum albumin in phosphate-buffered saline (PBS) for $30 \mathrm{~min}$ at room temperature and washed twice with PBS. Next, the samples were incubated with rabbit primary antibodies against mouse TLR2 (1:50; cat. no. ab16894), TLR9 (1:100; cat. no. ab37154) and NF-кB1 (1:100; cat. no. ab32360) (all from Abcam, Cambridge, MA, USA) overnight at $4^{\circ} \mathrm{C}$. Sections incubated with PBS without the primary antibody served as a negative control. Subsequently, sections were washed with PBS, and incubated with a polymer helper and polyclonal horseradish peroxidase-conjugated anti-rabbit IgG (Zhongshan Golden Bridge Biotechnology Co., Ltd., Beijing, China) for $1 \mathrm{~h}$ at $37^{\circ} \mathrm{C}$. Following counterstaining with hematoxylin, the samples were visualized under a light microscope (Carl Zeiss, Oberkochen, Germany). All data were analyzed using ImageJ software version 1.50i (National Institutes of Health, Bethesda, MD, USA).

Immunofluorescence staining for ATG5, ATG7, LC3, Beclin-1, mTOR and p62/SQSTM1 in rat IP tissues. For immunofluorescence examination of ATG5, ATG7, LC3, Beclin-1, mTOR and p62/SQSTM1 expression, slices were prepared according to the same procedure as described in the previous paragraph. Next, samples were incubated overnight at $4^{\circ} \mathrm{C}$ with mouse anti-ATG5 (1:250; cat. no. MAB5294), mouse anti-ATG7 (1:200; cat. no. MAB6608) (both from R\&D Systems Inc., Minneapolis, MN, USA), rabbit anti-LC3 (1:100; cat. no. 13394S; Cell Signaling Technology, Danvers, MA, USA), rabbit anti-Beclin-1 (1:200; ab210498; Abcam), mouse 

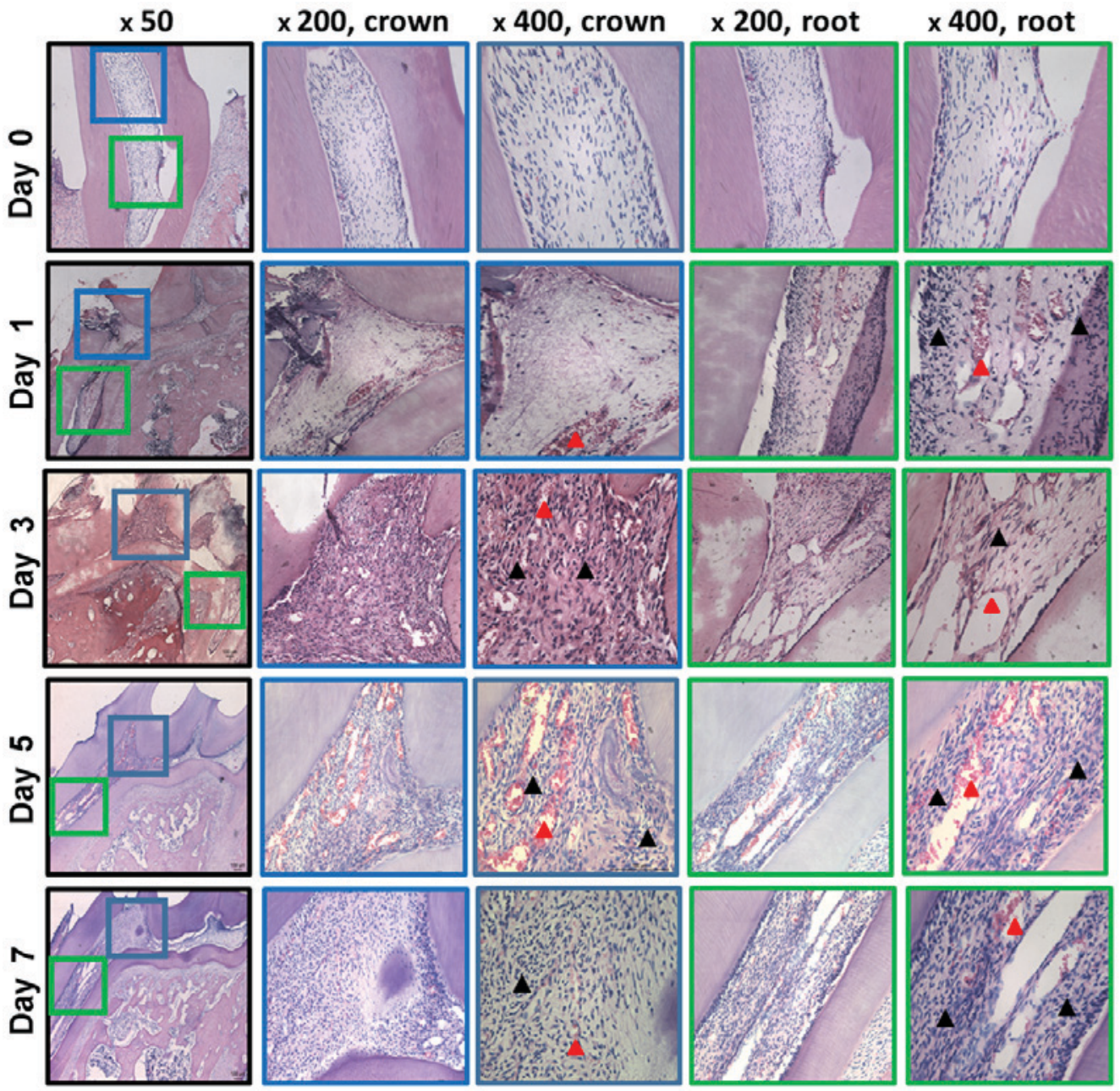

Figure 1. Histological analysis of the increase in inflammation between day 1 and 7. Hematoxylin and eosin was used for counterstaining. The blue frame displays the crown pulp, while the green frame displays the pulp. Black arrowheads indicate the odontoblast layer that displayed an irregular distribution, including vacuolar degeneration and a lack of odontoblasts. Dilated vessels and blood clot are indicated by the red arrowheads.

anti-p62/SQSTM1 (1:200; cat. no. ab56416; Abcam) and rabbit anti-mTOR (1:200; cat. no. ab2732; Abcam) primary antibodies in a PBS solution containing $1 \%$ bovine serum albumin at the recommended dilutions. The mouse anti-ATG7 and rabbit anti-LC3 antibodies were used for co-staining of the same section. Following further washes, sections were incubated for $30 \mathrm{~min}$ at $37^{\circ} \mathrm{C}$ with the Alexa Fluor 488-labeled goat anti-rabbit IgG secondary antibody exhibiting green fluorescence (1:200; Thermo Fisher Scientific, Inc., Waltham, MA, USA) or Cy3-labeled goat anti-mouse IgG exhibiting red fluorescence (1:200; Thermo Fisher Scientific, Inc.). Samples were washed again, stained with DAPI (Thermo Fisher Scientific, Inc.) for $5 \mathrm{~min}$ and then visualized under a confocal fluorescence microscope (Carl Zeiss). All data were analyzed using ImageJ software version 1.50i (National Institutes of Health).

Statistical analysis. All data are presented as the mean \pm standard error of the mean, and the data were analyzed using GraphPad Prism software, version 5.0 (GraphPad Software, Inc., La Jolla, CA, USA). The statistical significance of differences between the D0 and IP groups was assessed using one-way analysis of variance. All experiments were independently repeated in triplicate. $\mathrm{P}<0.05$ was considered to denote a statistically significant difference.

\section{Results}

$H \& E$ staining of rat IP tissues. In the D0 group, no inflammation was visible in the dental pulp tissue, and the dental pulp cells and vessels exhibited normal shapes and distributions. The dental pulp cells were star-shaped, with intense nuclear staining and light uniform staining in the cytoplasm. Peripherally, a closely spaced odontoblast layer was present, with the cell bodies exhibiting a typical cylindrical shape. The odontoblast layer appeared normal within the radicular pulp tissue (Fig. 1).

In the IP group, the H\&E staining revealed an increase in inflammation between D1 and D7 (Fig. 1). The exposed pulp tissue displayed a striking increase in hyperemic dilated vessels, inflammatory cell infiltration and a disrupted odontoblast layer, particularly at D5 and D7. An increasing number of neutrophils accumulated below the exposed point in the coronal pulp tissue. At D5 and D7, an increased number of hyperemic dilated vessels were observed in the radicular and coronal pulp, and a large number of red blood cells also appeared (Fig. 1, arrowheads). Notably, the degree of inflammation in the radicular pulp promoted the formation of hyperemic dilated vessels and neutrophil granulocyte infiltration. Based on these histological features, IP occurred 

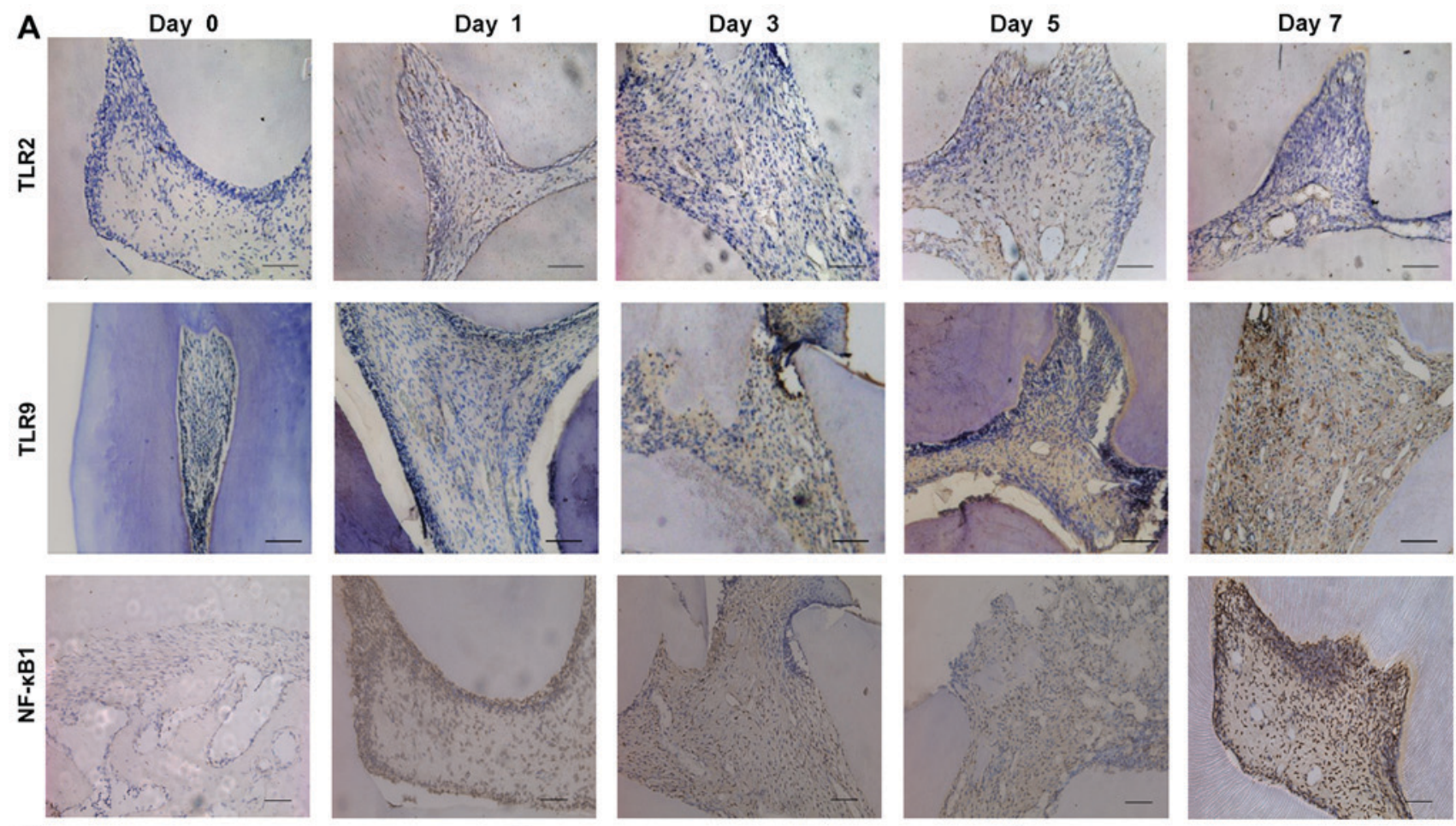

B

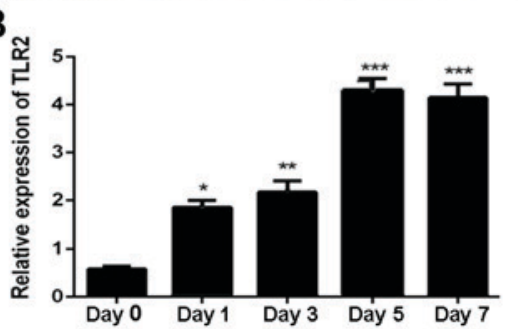

C

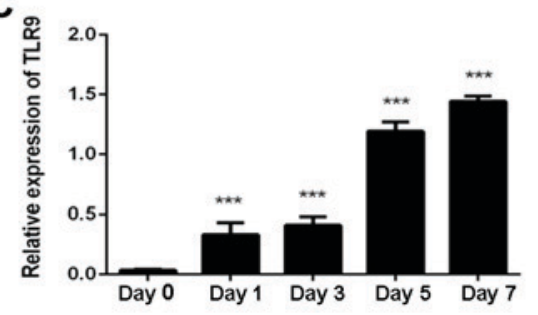

$\mathbf{D}_{\infty}$

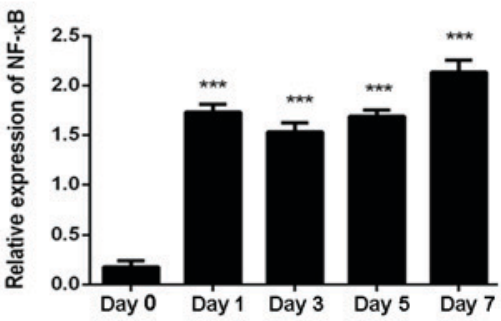

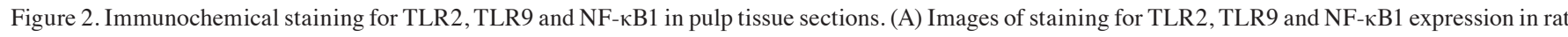
IP tissues (scale bar, $100 \mu \mathrm{m}$ ). (B) TLR2, (C) TLR9 and (D) NF-кB1 expression levels were significantly upregulated in the IP group on days 1-7 as compared with the day 0 (control) group. The experiments were repeated in triplicate, and the results were analyzed using ImageJ software. Error bars represent the standard error of the mean. ${ }^{*} \mathrm{P}<0.05,{ }^{* *} \mathrm{P}<0.01$ and ${ }^{* * *} \mathrm{P}<0.001$ vs. day 0 . TLR, toll-like receptors; NF- $\kappa \mathrm{B} 1$, nuclear factor- $\kappa \mathrm{B} 1$; IP, irreversible pulpitis.

in rats at D1 following surgery, and dental pulp injury caused a severe 7-day period of pulp inflammation in the IP group. Furthermore, inflammation increased in a time-dependent manner, and the inflammation occurred gradually from the coronal to the radicular pulp.

Expression levels of TLR2, TLR 9 and NF- $\kappa B 1$ in rat IP tissues. Pulp inflammation evidently altered the expression levels of TLR2, TLR9 and NF-kB1. As shown in Fig. 2A, TLR2, TLR9 and NF- $\mathrm{KB} 1$ expression was observed in the odontoblast layers, odontoblast processes in the tubules of the predentin, stromal pulp fibroblast-like cells and vessel endothelial-like cell lining of the pulpitis tissues in the IP group between D1 and D7. The immunohistochemical staining revealed significantly higher levels of TLR2 in the IP group as compared with that observed in the D0 control group. According to the measurement of the TLR2-expressing area, a gradual increase in TLR2 expression between D1 and D7 was detected, and TLR2 levels peaked at D5 and D7 post-surgery (Fig. 2B). Based on these results, TLR2 expression was upregulated in the rat pulpitis tissue, and the degree of inflammation was increased in a time-dependent manner. In addition, TLR9 expression displayed a similar pattern to TLR2, and was gradually upregulated between
D1 and D7 (Fig. 2C). NF-кB1 was also expressed at high levels between D1 and D7 in the tissues of IP rats, with peak expression observed at D7 (Fig. 2D). Thus, inflammation was increased in rat pulpitis tissues in a time-dependent manner.

Expression levels of ATGs, Beclin-1, mTOR and p62/SQSTM1 in rat coronal pulpitis tissues. Immunofluorescence staining was subsequently used to investigate the expression of autophagy-associated proteins in IP tissues, and the results revealed that ATG5, ATG7, LC3 and Beclin-1 were highly expressed in the rat coronal pulpitis tissues. In D0 rat coronal pulp tissues, ATG5 was expressed at low levels in the odontoblast layers and vessel endothelial cells. However, intense ATG5 staining was observed in the vessel endothelial cells, odontoblast layers, interstitial connective tissues and infiltrating inflammatory cells of the IP group at different time points (Fig. 3A). The relative level of ATG5 expression in the IP tissue was significantly increased as compared with that in the D0 control group $(\mathrm{P}<0.001$; Fig. 3B). In the rat coronal pulp, ATG5 expression increased immediately on D1 and remained at high levels between D3 and D7 (Fig. 3B). The expression patterns of ATG7, LC3 and Beclin-1 were similar to that of ATG5 expression (Fig. 3C-E). Thus, ATG (ATG5, 
A
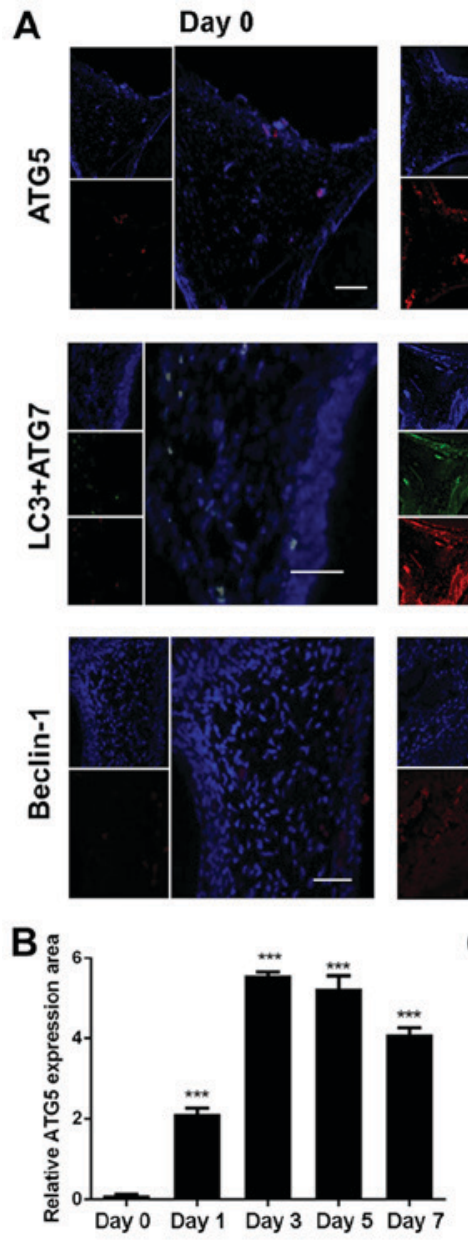

Day 1
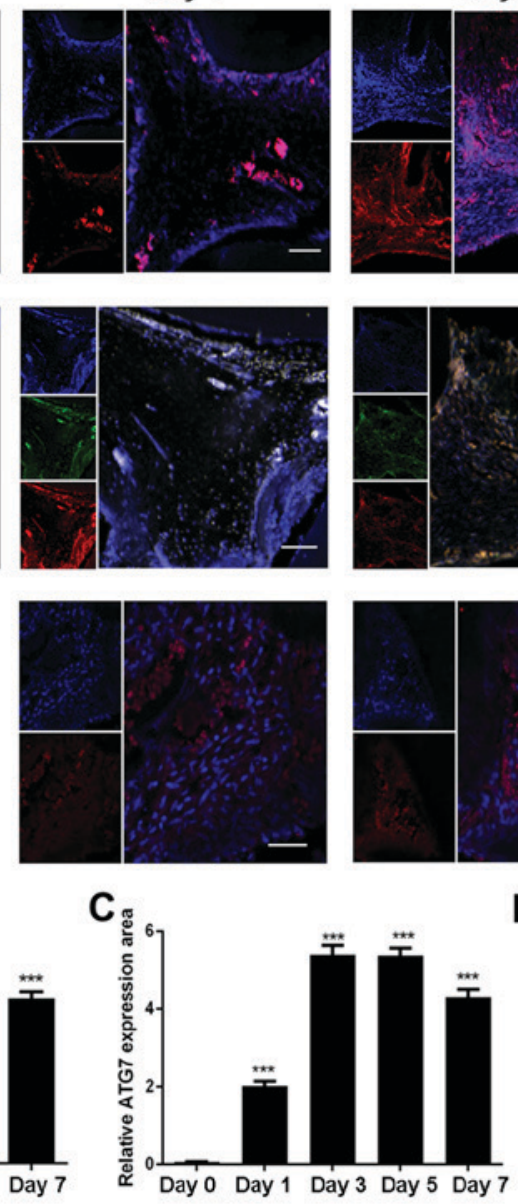

Day 3
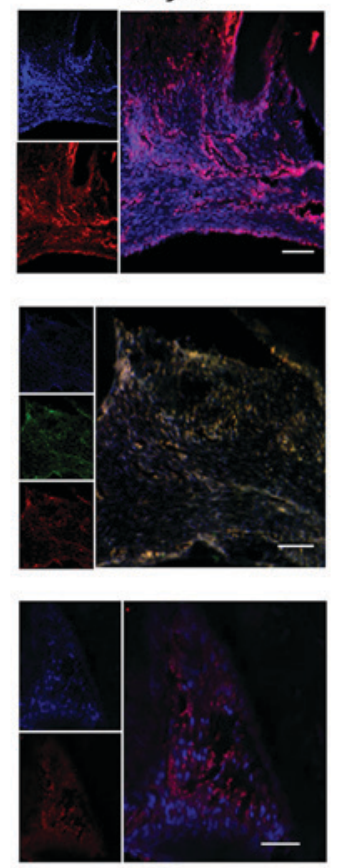

$D_{s}$

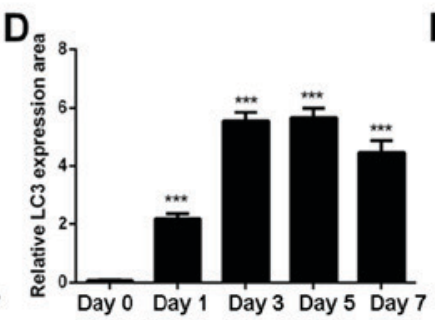

Day 5
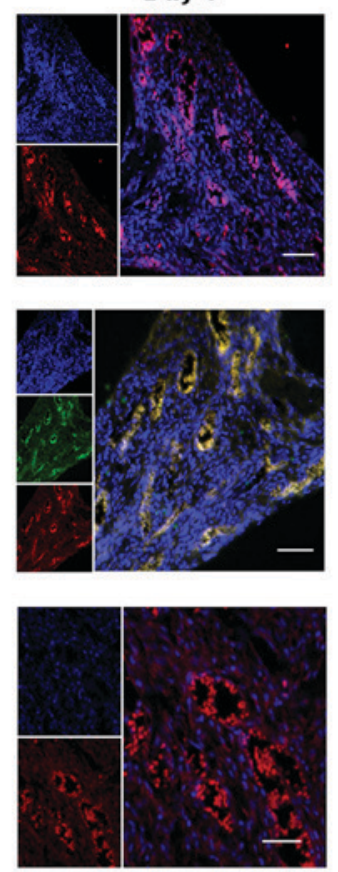

Day 7
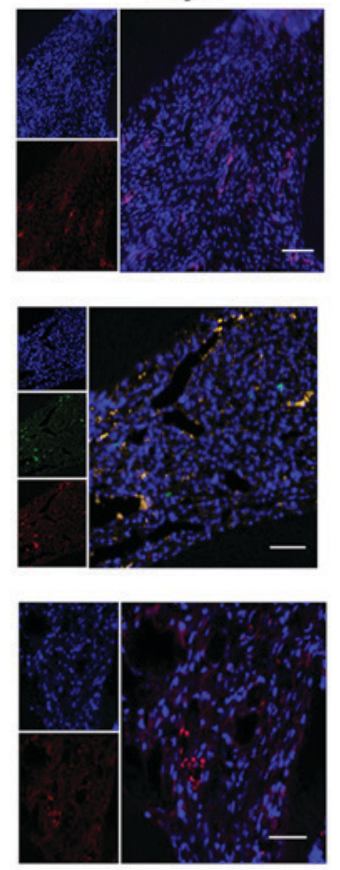

Figure 3. ATG5, ATG7, LC3 and Beclin-1 expression in the coronal pulp. (A) Images of the immunofluorescence staining for ATG5 (red), ATG7 (red), LC3 (green), Beclin-1 (red) and DAPI (blue) were captured using a fluorescence microscope in the control (day 0) and IP group (days 1-7; magnification, x200; scale bar, $100 \mu \mathrm{m}$ ). (B) ATG5, (C) ATG7, (D) LC3 and (E) Beclin-1 expression area was significantly increased in the IP group on days 1-7 as compared with the day 0 group. The experiments were repeated in triplicate, and the results were analyzed using ImageJ software. Error bars represent the standard error of the mean. ${ }^{* * * *} \mathrm{P}<0.001$ vs. day 0 . ATG, autophagy-related; LC3, light chain 3; IP, irreversible pulpitis.

ATG7 and LC3) and Beclin-1 expression was upregulated in the rat coronal pulpitis tissues between D1 and D7, consistent with the progression of inflammation in the rat coronal pulpitis tissue.

Furthermore, as observed in Fig. 4, the expression levels of mTOR and p62/SQSTM1 was evidently decreased between D1 and D7, with a significant difference observed at D5 and D7 compared with the D0 group. These findings indicated that autophagy was activated in the present study (Fig. 4).

\section{Expression levels of ATGs, Beclin-1, mTOR and p62/SQSTM1} in radicular pulpitis tissues. In the D0 group, ATG5 was expressed at low levels in the odontoblast layers and vessel endothelial cells of the radicular pulp, while higher levels of ATG5 expression were observed in the odontoblast layers and vessel endothelial cells of the radicular pulpitis tissues (Fig. 5A). Between D3 and D7, statistically significant increases in ATG5 expression were observed in the pulpitis tissue when compared with the D0 group (Fig. 5B; P<0.001). The expression pattern of ATG5 in the root pulpitis tissue differed from that in coronal pulpitis tissue, since ATG5 was gradually upregulated in a time-dependent manner and peaked at D7 in radicular tissues (Fig. 5B), whereas it peaked at D3 and then slightly decreased in coronal tissues. ATG7, LC3 and Beclin-1 expression levels also increased gradually from D1 onward, and the expression patterns of these proteins was similar to that of ATG5 (Fig. 5C-E). Furthermore, the expression levels of mTOR and p62/SQSTM1 decreased gradually between D1 and D7, with a significant difference observed at D7 compared with the D0 group, suggesting that autophagy was activated (Fig. 6). Based on these results, it can be speculated that increased expression of autophagy-associated proteins may be involved in the regulation of inflammatory progression in rat tooth pulp.

\section{Discussion}

Autophagy has been recognized as an important regulator of numerous biological processes. Autophagy has multiple roles in infection, including the direct elimination of microorganisms, the regulation of inflammation and the interception of invading pathogens (16). Mice that lack the essential autophagy genes ATG7 or ATG5 present spontaneous sterile lung inflammation (13). The study of autophagy in order to improve the understanding of human diseases has received growing attention (27). Autophagy has recently been demonstrated to 

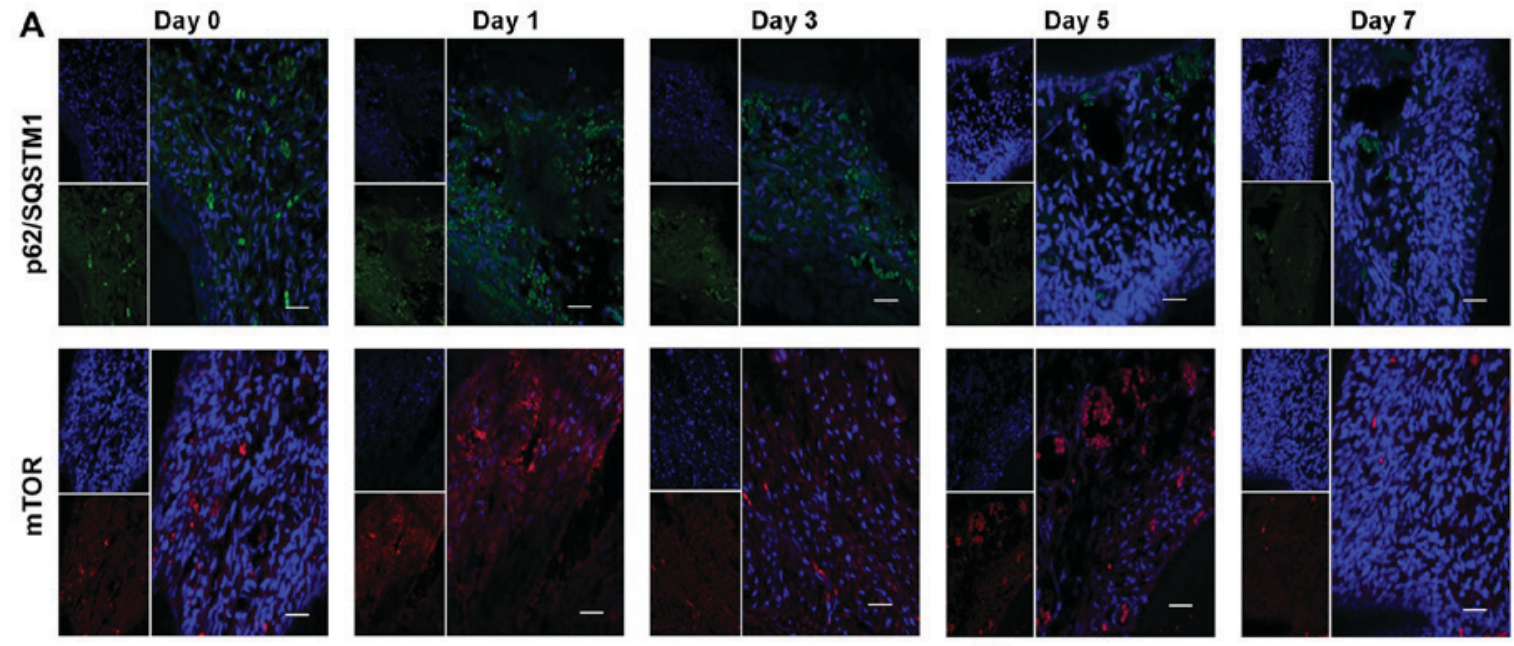

B

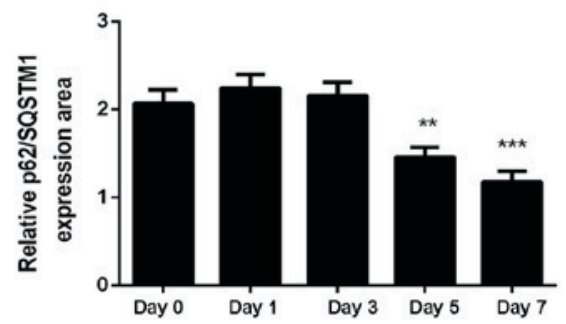

C

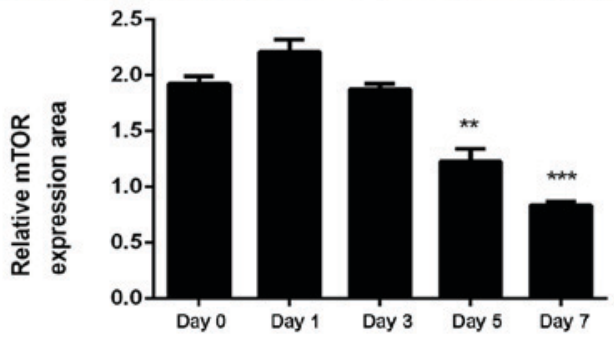

Figure 4. mTOR and p62/SQSTM1 expression in the coronal pulp. (A) Images of immunofluorescence staining for mTOR (red), p62/SQSTM1 (green) and DAPI (blue) were captured using a fluorescence microscope (magnification, x200; scale bar, $100 \mu \mathrm{m}$ ). (B) p62/SQSTM1 and (C) mTOR were significantly decreased in the irreversible pulpitis group on days 5 and 7 as compared with the day 0 group. The experiments were repeated in triplicate, and the results were analyzed using ImageJ software. Error bars represent the standard error of the mean. ${ }^{* *} \mathrm{P}<0.01$ and ${ }^{* * * *} \mathrm{P}<0.001$ vs. day 0 group. mTOR, mammalian target of rapamycin; SQSTM1, sequestosome 1.

A
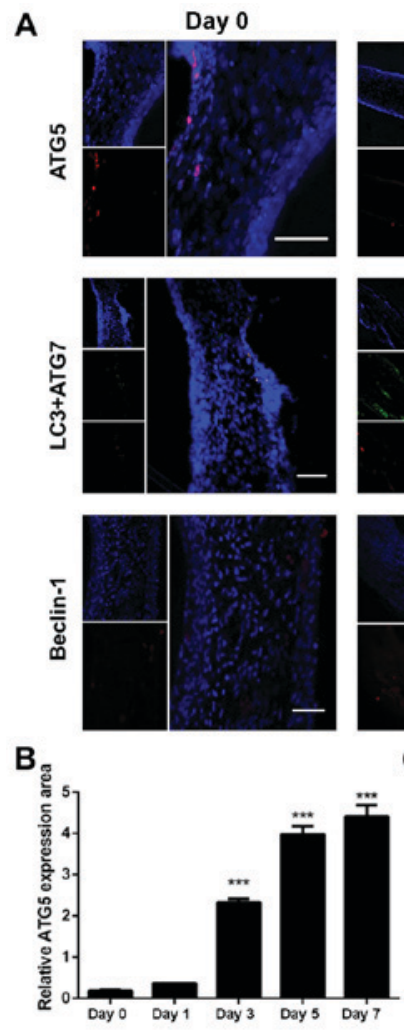

Day 1
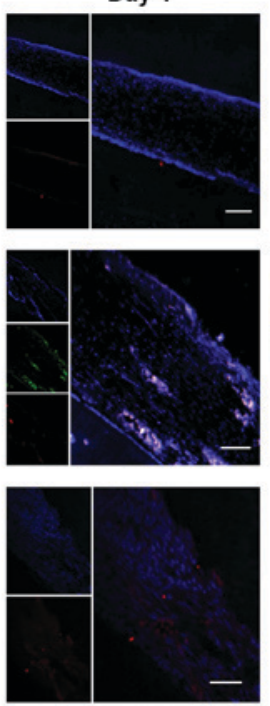

C
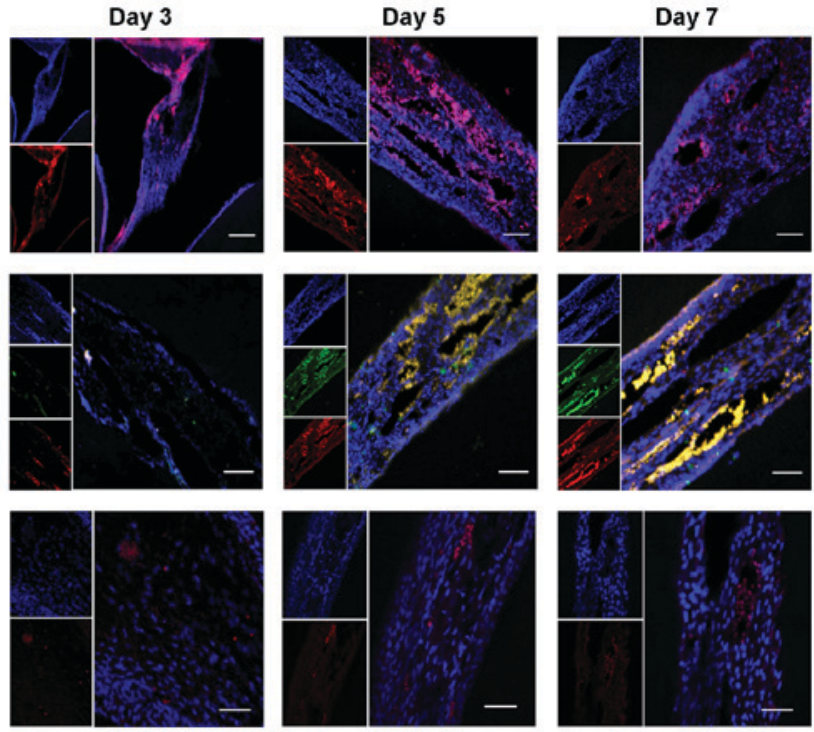

$E_{8}$
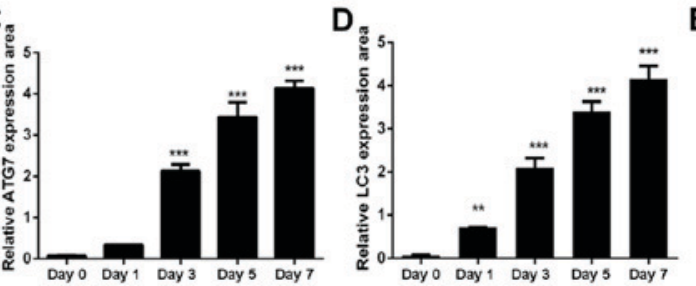

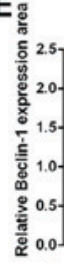

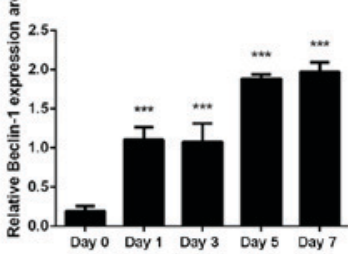

Figure 5. ATG5, ATG7, LC3 and Beclin-1 expression in the radicular pulp. (A) Images of the immunofluorescence staining for ATG5 (red), ATG7 (red), LC3 (green), Beclin-1 (red) and DAPI (blue) were captured using a fluorescence microscope (days 1-7; magnification, x200; scale bar, 100 $\mu \mathrm{m}$ ). (B) ATG5, (C) ATG7, (D) LC3 and (E) Beclin-1 expression area was significantly increased in the irreversible pulpitis group on days 1-7 as compared with the day 0 group. The experiments were repeated in triplicate, and the results were analyzed using ImageJ software. Error bars represent the standard error of the mean. ${ }^{* *} \mathrm{P}<0.01$ and ${ }^{* * *} \mathrm{P}<0.001$ vs. day 0 . ATG, autophagy-related; LC3, light chain 3 . 

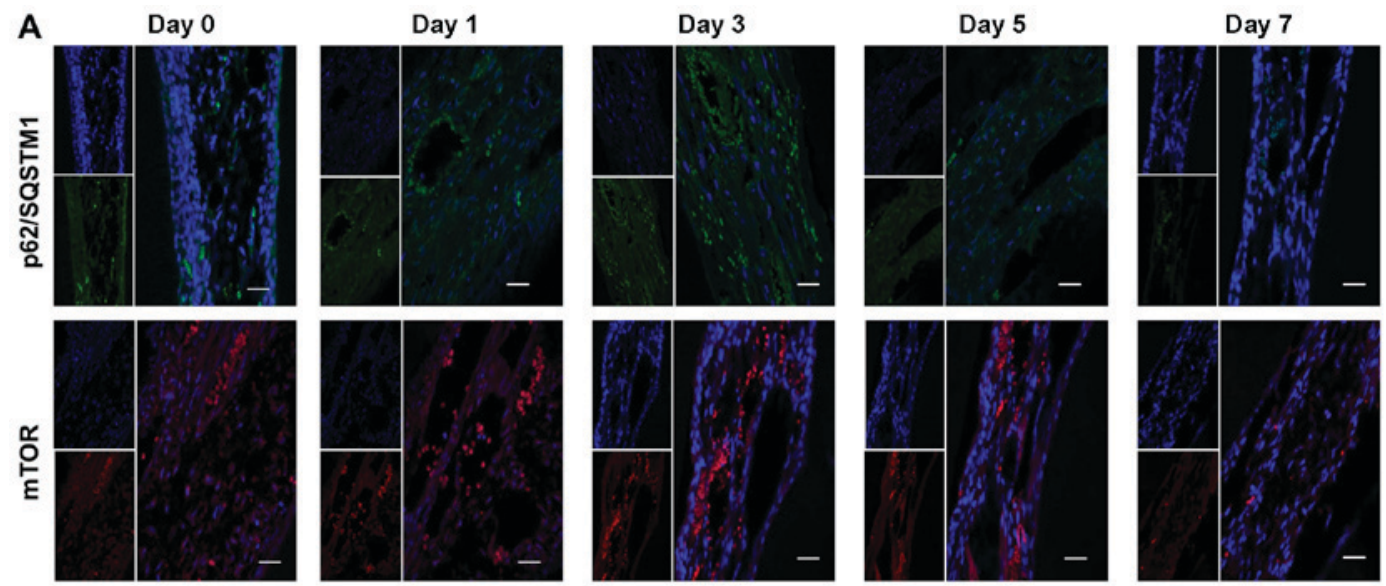

B

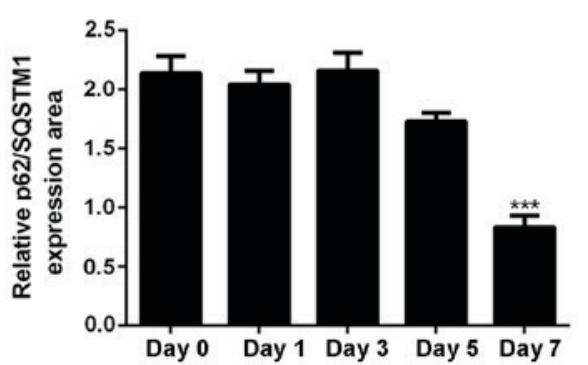

C

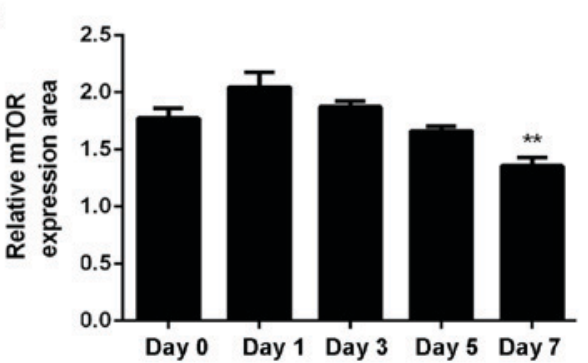

Figure 6. mTOR and p62/SQSTM1 expression in the radicular pulp. (A) Images of immunofluorescence staining for mTOR (red), p62/SQSTM1 (green) and DAPI (blue) were captured using a fluorescence microscope (magnification, x200; scale bar, $100 \mu \mathrm{m}$ ). (B) p62/SQSTM1 and (C) mTOR were significantly decreased in the irreversible pulpitis group on day 7 as compared with the day 0 group. The experiments were repeated in triplicate, and the results were analyzed using ImageJ software. Error bars represent the standard error of the mean. ${ }^{* * *} \mathrm{P}<0.01$ and ${ }^{* * * *} \mathrm{P}<0.001$ vs. day 0 group. mTOR, mammalian target of rapamycin; SQSTM1, sequestosome 1.

serve a crucial role in the defense against dental pulpitis cell inflammation in vitro (7). However, the roles of autophagy in IP in vivo are not currently well understood. Therefore, the present study explored the expression levels of various ATGs in IP tissues in vivo using immunofluorescence staining to help elucidate the IP pathogenesis.

Pulp exposure is typically used to observe the natural progression of IP (28). In the current study, a rat IP model was successfully constructed using this method. It has been reported that TLRs and NF- $\mathrm{KB} 1$ are expressed at high levels in pulpitis tissue (7). The present study results of $H \& E$ staining, and analyses of TLR and NF- $\mathrm{kB} 1$ expression levels revealed that the exposed pulp, without the protection of enamel, was vulnerable to bacterial invasion and developed pulpitis. The pathogenesis and histological expression patterns differ between reversible and irreversible pulpitis. IP is characterized by exposure of the pulp with considerable inflammatory cell infiltration or even necrosis in the coronal and root pulp in a time-dependent manner, while reversible pulpitis is characterized by hyperemic vessels and mild inflammatory cell infiltration in the coronal pulp $(29,30)$. The current study utilized a rat IP model, and thus the expression of autophagy-associated proteins in reversible pulpitis in vivo requires further exploration.

Autophagic flux is considered to reflect the level of autophagy activity $(8,31)$. ATGs, Beclin- 1 and LC3, which are essential in autophagosome formation, have been widely used as autophagy markers. In the present study, the expression levels of ATGs, Beclin-1 and LC3 were found to be upregulated. However, these proteins reflect the formation of autophagosomes, but not the level of autophagy activity. LC3 only reflects the level of autophagic flux in combination with LC3 turnover (31). LC3 includes LC3-I and LC3-II; LC3-II is the lipidated form of LC3-I that is present in the inner and outer membranes of the autophagosome. LC3-II is expressed at high levels as the level of autophagic flux increases, while LC3-I is expressed at lower levels; thus, the ratio of LC3-II/LC3-I is used as a marker of autophagic flux. Although LC3-II/LC3-I is a useful indicator in biochemical assays, it was not used in the fluorescence microscopy assays in the present study. However, p62/SQSTM1 selectively interacts with LC3 to induce autophagosome formation and is efficiently degraded during autophagy; thus, p62/SQSTM1 is an indicator of autophagic flux, and an inverse correlation has been observed between its expression levels and autophagy activity $(11,32)$. Furthermore, mTOR is a known suppressor of autophagy (33), and its expression negatively is correlated with autophagy activity; thus, the expression of mTOR serves as a marker of autophagy activation. In the present study, p62/SQSTM1 and mTOR were found to be expressed at low levels, suggesting that autophagy was activated in IP tissues.

Autophagy activity is increased during inflammation (34) and has been proven to be involved in the dental pulp cell response to LPS in vitro and in periapical lesions in vivo $(35,36)$. In the present study, autophagy occurred in IP tissues and was consistent with the progression of inflammation, implying that autophagy may be involved in the dental IP pathology (36). In addition, the area of tissue expressing autophagy markers increased in a time-dependent manner from the coronal to radicular pulp. However, the expression pattern varied between 
the coronal and radicular pulpitis tissues. Autophagy expression in the coronal pulpitis tissue occurred earlier compared with in the radicular pulpitis tissue. Thus, autophagy occurs in a manner consistent with the pathological progression of inflammation in the dental pulp.

The expression of autophagy-associated proteins was mainly upregulated in the rat odontoblast layer and microvascular endothelial cells in the IP tissues in vivo. The odontoblast layer serves a key role in controlling pulpitis. When odontoblasts are damaged, the dental pulp stem cells (DPSCs) migrate and differentiate into odontoblast-like cells, and form tertiary dentin $(18,37,38)$. Autophagy regulates odontoblast differentiation when DPSCs respond to LPS stimulation in vitro (7), which explains why autophagy was activated in the odontoblast layer in IP tissues. In response to inflammation, autophagy also contributes to vascular remodeling by regulating cell adhesion molecules, such as intercellular adhesion molecule-1 and vascular cell adhesion protein-1 (39). Based on these findings, autophagy may serve important roles in pulpitis pathogenesis and vascular remodeling in IP tissues in vitro, and the current study results indicated a similar role for autophagy in IP in vivo.

Autophagy inhibits TLR expression and inflammation (16), while TLRs also induce autophagy and inflammation (40-42). In the present study, the expression of autophagy-associated proteins was increased in IP tissues in a time-dependent manner, and a similar increasing trend was observed for TLR expression. Nevertheless, with the increase in the expression of autophagy-associated proteins, dental pulp inflammation was not substantially improved at D7 postsurgery, suggesting that inflammation had progressed in IP tissues. Therefore, it is hypothesized that autophagy activation in IP tissues depends on the TLR-mediated increase in inflammation. Further studies that examine this hypothesis are required in the future.

In conclusion, the expression levels of autophagy-associated proteins (ATG5, ATG7, LC3 and Beclin-1) were demonstrated to be upregulated and the expression levels of mTOR and p62/SQSTM1 were downregulated, indicating that autophagy was activated in rat IP tissues. These results suggested that autophagy may have an important role in IP, and future studies will focus on the biological role of autophagy in IP and may provide a new treatment strategy for dental endodontics.

\section{Acknowledgements}

Not applicable.

\section{Funding}

This study was supported by grants from the National Natural Science Foundation of China (grant no. 81500806), the China Postdoctoral Science Foundation (grant no. 2015M581633), the Shanghai Pujiang Program (grant no. 16PJ1408400), and the Fundamental Research Funds for the Central Universities (grant nos. 2014KJ090, 1501219191 and 1501219115).

\section{Availability of data and materials}

All data generated or analyzed during the present study are included in this published article.

\section{Authors' contributions}

CC and YX conceived the study. SQ, JQ, FC, FT, YZ and GS designed the study and performed the experiments. SQ, JQ, PZ, JY and SG performed data analysis and interpretation. SQ and JQ drafted the manuscript. FC and YX revised the manuscript for important intellectual content. All the authors agreed to be accountable for all aspects of the work in ensuring that questions related to the accuracy or integrity of any part of the work are appropriately investigated and resolved. All the authors approved the final version to be submitted to the journal. CC gave the final approval of the version to be published.

\section{Ethical approval and consent to participate}

The study was approved by the Ethics Committee of Shanghai Tenth People's Hospital, Tongji University School of Medicine (Shanghai, China; approval no. SHDSYY-2016-0302).

\section{Patient consent for publication}

Not applicable.

\section{Competing interests}

The authors declare that they have no competing interests.

\section{References}

1. Pan H, Cheng L, Yang H, Zou W, Cheng $\mathrm{R}$ and $\mathrm{Hu} \mathrm{T}$ : Lysophosphatidic acid rescues human dental pulp cells from ischemia-induced apoptosis. J Endod 40: 217-222, 2014.

2. Ferraz CC, Henry MA, Hargreaves KM and Diogenes A: Lipopolysaccharide from Porphyromonas gingivalis sensitizes capsaicin-sensitive nociceptors. J Endod 37: 45-48, 2011.

3. Akira S, Takeda K and Kaisho T: Toll-like receptors: Critical proteins linking innate and acquired immunity. Nat Immunol 2: 675-680, 2001

4. Mutoh N, Tani-Ishii N, Tsukinoki K, Chieda K and Watanabe K: Expression of toll-like receptor 2 and 4 in dental pulp. J Endod 33: 1183-1186, 2007.

5. Omiya S, Omori Y, Taneike M, Protti A, Yamaguchi O, Akira S, Shah AM, Nishida K and Otsu K: Toll-like receptor 9 prevents cardiac rupture after myocardial infarction in mice independently of inflammation. Am J Physiol Heart Circ Physiol 311: H1485-H1497, 2016.

6. Dias AA, Silva CO, Santos JP, Batista-Silva LR, Acosta CC, Fontes AN, Pinheiro RO, Lara FA, Machado AM, Nery JA, et al: DNA sensing via TLR-9 constitutes a major innate immunity pathway activated during erythema nodosum leprosum. J Immunol 197: 1905-1913, 2016.

7. Pei F, Wang HS, Chen Z and Zhang L: Autophagy regulates odontoblast differentiation by suppressing NF- $\kappa \mathrm{B}$ activation in an inflammatory environment. Cell Death Dis 7: e2122, 2016.

8. Mathiassen SG, De Zio D and Cecconi F: Autophagy and the cell cycle: A complex landscape. Front Oncol 7: 51, 2017.

9. Pradhan AK, Talukdar S, Bhoopathi P, Shen XN, Emdad L, Das SK, Sarkar D and Fisher PB: mda-7/IL-24 mediates cancer cell-specific death via regulation of miR-221 and the beclin-1 axis. Cancer Res 77: 949-959, 2017.

10. Klionsky DJ, Baehrecke EH, Brumell JH, Chu CT, Codogno P, Cuervo AM, Debnath J, Deretic V, Elazar Z, Eskelinen EL, et al: A comprehensive glossary of autophagy-related molecules and processes (2nd edition). Autophagy 7: 1273-1294, 2011.

11. Lamark T, Svenning S and Johansen T: Regulation of selective autophagy: The p62/SQSTM1 paradigm. Essays Biochem 61: 609-624, 2017.

12. Larsen KB, Lamark T, Øvervatn A, Harneshaug I, Johansen T and Bjorkoy G: A reporter cell system to monitor autophagy based on p62/SQSTM1. Autophagy 6: 784-793, 2010. 
13. Abdel Fattah E, Bhattacharya A, Herron A, Safdar Z and Eissa N Critical role for IL-18 in spontaneous lung inflammation caused by autophagy deficiency. J Immunol 194: 5407-5416, 2015.

14. Deretic V: Autophagy in immunity and cell-autonomous defense against intracellular microbes. Immunol Rev 240: 92-104, 2011.

15. Shi CS, Shenderov K, Huang NN, Kabat J, Abu-Asab M, Fitzgerald KA, Sher A and Kehrl JH: Activation of autophagy by inflammatory signals limits IL- $1 \beta$ production by targeting ubiquitinated inflammasomes for destruction. Nat Immunol 13: 255-263, 2012

16. Deretic V, Saitoh T and Akira S: Autophagy in infection, inflammation and immunity. Nat Rev Immunol 13: 722-737, 2013.

17. Huang HY, Wang WC, Lin PY, Huang CP, Chen CY and Chen YK: The roles of autophagy and hypoxia in human inflammatory periapical lesions. Int Endod J 51 (Suppl 2): e125-e145, 2018.

18. Farges J, Alliot-Licht B, Baudouin C, Msika P, Bleicher F and Carrouel F: Odontoblast control of dental pulp inflammation triggered by cariogenic bacteria. Front Physiol 4: 326, 2013.

19. Deretic V: Autophagy as an innate immunity paradigm: Expanding the scope and repertoire of pattern recognition receptors. Curr Opin Immunol 24: 21-31, 2012.

20. Konisti S, Kiriakidis S and Paleolog EM: Hypoxia-a key regulator of angiogenesis and inflammation in rheumatoid arthritis. Nat Rev Rheumatol 8: 153-162, 2012.

21. Mazure NM and Pouysségur J: Hypoxia-induced autophagy: Cell death or cell survival? Curr Opin Cell Biol 22: 177-180, 2010.

22. Vellaisamy K, Li G, Ko CN, Zhong HJ, Fatima S, Kwan HY, Wong CY, Kwong WJ, Tan W, Leung CH and Ma DL: Cell imaging of dopamine receptor using agonist labeling iridium (iii) complex. Chem Sci 9: 1119-1125, 2017.

23. Liu LJ, Wang W, Huang SY, Hong Y, Li G, Lin S, Tian J, Cai Z Wang HD, Ma DL and Leung CH: Inhibition of the Ras/Raf interaction and repression of renal cancer xenografts in vivo by an enantiomeric iridium(iii) metal-based compound. Chem Sci 8: 4756-4763, 2017.

24. Wang W, Vellaisamy K, Li G, Wu C, Ko CN, Leung CH and Ma DL: Development of a long-lived luminescence probe for visualizing $\beta$-galactosidase in ovarian carcinoma cells. Anal Chem 89: 11679-11684, 2017.

25. Hori Y, Otomura N, Nishida A, Nishiura M, Umeno M, Suetake I and Kikuchi K: Synthetic-molecule/protein hybrid probe with fluorogenic switch for live-cell imaging of DNA methylation. J Am Chem Soc 140: 1686-1690, 2018.

26. Wang H, Feng Z, Del Signore SJ, Rodal AA and Xu B: Active probes for imaging membrane dynamics of live cells with high spatial and temporal resolution over extended time scales and areas. J Am Chem Soc 140: 3505-3509, 2018

27. Rubinsztein DC, Codogno P and Levine B: Autophagy modulation as a potential therapeutic target for diverse diseases. Nat Rev Drug Discov 11: 709-730, 2012.

28. Wang Y, Zhai S, Wang H, Jia Q, Jiang W, Zhang X, Zhang A, Liu J and Ni L: Absent in melanoma 2 (AIM2) in rat dental pulp mediates the inflammatory response during pulpitis. J Endod 39: 1390-1394, 2013.
29. Gibbs JL, Urban R and Basbaum AI: Paradoxical surrogate markers of dental injury-induced pain in the mouse. Pain 154: 1358-1367, 2013.

30. Ricucci D, Siqueira JF Jr, Loghin S and Lin LM: Pulp and apical tissue response to deep caries in immature teeth: A histologic and histobacteriologic study. J Dent 56: 19-32, 2017.

31. Mizushima N, Yoshimorim T and Levine B: Methods in mammalian autophagy research. Cell 140: 313-326, 2010.

32. Klionsky DJ, Abdalla FC, Abeliovich H, Abraham RT, Acevedo-Arozena A, Adeli K, Agholme L, Agnello M, Agostinis P, Aguirre-Ghiso JA, et al: Guidelines for the use and interpretation of assays for monitoring autophagy. Autophagy 8: 445-544, 2012.

33. Chen WR, Liu HB, Chen YD, Sha Y, Ma Q, Zhu PJ and Mu Y: Melatonin attenuates myocardial ischemia/reperfusion injury by inhibiting autophagy via an AMPK/mTOR signaling pathway. Cell Physiol Biochem 47: 2067-2076, 2018.

34. Li L, Zhu YQ, Jiang L and Peng W: Increased autophagic activity in senescent human dental pulp cells. Int Endod J 45: 1074-1079, 2012.

35. Pei F, Lin H, Liu H, Li L, Zhang L and Chen Z: Dual role of autophagy in lipopolysaccharide-induced preodontoblastic cells. J Dent Res 94: 175-182, 2015.

36. Zhu L, Yang J, Zhang J and Peng B: The presence of autophagy in human periapical lesions. J Endod 39: 1379-1384, 2013.

37. Kenmotsu M, Matsuzaka K, Kokubu E, Azuma T and Inoue T: Analysis of side population cells derived from dental pulp tissue. Int Endod J 43: 1132-1142, 2010.

38. Matsuoka K, Matsuzaka K, Yoshinari M and Inoue T: Tenascin-C promotes differentiation of rat dental pulp cells in vitro. Int Endod J 46: 30-39, 2013

39. Duan J, Yu Y, Yu Y, Li Y, Huang P, Zhou X, Peng S and Sun Z: Silica nanoparticles enhance autophagic activity, disturb endothelial cell homeostasis and impair angiogenesis. Part Fibre Toxicol 11: 50, 2014

40. Anand PK, Tait SW, Lamkanfi M, Amer AO, Nunez G, Pagès G, Pouysségur J, McGargill MA, Green DR and Kanneganti TD: TLR2 and RIP2 pathways mediate autophagy of Listeria monocytogenes via extracellular signal-regulated kinase (ERK) activation. J Biol Chem 286: 42981-42991, 2011.

41. Henault J, Martinez J, Riggs JM, Tian J, Mehta P, Clarke L, Sasai M, Latz E, Brinkmann MM, Iwasaki A, et al: Noncanonical autophagy is required for type I interferon secretion in response to DNA-immune complexes. Immunity 37 : 986-997, 2012.

42. Xu Y, Jagannath C, Liu XD, Sharafkhaneh A, Kolodziejska KE and Eissa NT: Toll-like receptor 4 is a sensor for autophagy associated with innate immunity. Immunity 27: 135-144, 2007.

This work is licensed under a Creative Commons Attribution-NonCommercial-NoDerivatives 4.0 International (CC BY-NC-ND 4.0) License. 